\title{
Möglichkeiten und Grenzen netzbasierter Lehre in den Rechtswissenschaften
}

\author{
Stephan Lorenz*
}

\section{A. Das Lernziel}

Was und wie lernt ein angehender Jurist - und was kann „das Internet“ dafür tun? Das Jurastudium ist ein wissenschaftliches Studium, das zu einer beruflichen Qualifikation führen soll. Man zählt die Juristischen Fakultäten daher auch zu den sogenannte „Professionsfakultäten“. Das Recht ist also gerade in der Praxis eine wissenschaftliche Disziplin. Ziel des Studiums ist nicht der Erwerb unstrukturierter Kenntnisse von Gesetzen und Fällen, sondern das Erlernen des „wissenschaftlichen Handwerks“. Richtige Rechtsanwendung verlangt also nicht nur Kenntnis von Rechtsnormen, Subsumtionstechnik, Definitionen und Verfahrensabläufen, sondern auch und vor allem wissenschaftliche Methoden. ${ }^{1}$ Wichtigstes Mittel für den Juristen ist - welchen Beruf auch immer er ergreifen mag - die Sprache. Nicht nur juristisches Verständnis, sondern auch juristische Argumentation lebt von einer klaren Gliederung der Gedanken. Ein guter Jurist zeichnet sich durch den „roten Faden“ eines klaren juristischen Gedankengangs aus. Dazu bedarf es der Fähigkeit, komplexe Rechtsprobleme zu analysieren und in juristische Einzelprobleme zu gliedern, diese einer Lösung zuzuführen und anschließend wieder in den Gesamtkontext zu stellen.

\section{B. Die Lehr-und Lernmethode}

Die meisten Jura-Erstsemester werden keine besonderen Vorkenntnisse vom Recht und seiner Funktionsweise haben. Jura lernt man zunächst anhand einer (Anfänger-)Vorlesung, die von Arbeitsgemeinschaften in Kleingruppen begleitet werden sollte. Unabdingbar ist aber auch das Selbststudium in Form der Lektüre von Lehrbüchern und der Anwendung des Gelernten anhand von Übungsfällen. Ein erfolgreiches Staatsexamen setzt voraus, dass der in Komplexität und Masse nicht unerhebliche Stoff mehrmals durchgearbeitet, durchgedacht und vor allem auch geübt wird. Das setzt ein hohes Maß an Zeit und Selbstdisziplin voraus. Der normale Examenskandidat dürfte bis zur Ersten Juristischen Staatsprüfung den Prüfungsstoff in der Regel mindestens drei Mal durchgegangen sein, um ihn in klausurtauglicher Weise verinnerlicht zu haben. Daneben muss Klausurtechnik geübt werden, das

* Der Autor ist Inhaber eines Lehrstuhls für Bürgerliches Recht, Internationales Privatrecht und Rechtsvergleichung an der LMU München und Mitglied des Bayerischen Verfassungsgerichtshofs. Er ist Preisträger des Ars legendi-Preises für exzellente Hochschullehre 2013.

1 Unentbehrlich sind dabei natürlich auch die Grundlagen des Rechts und sein Verhältnis zu den Nachbargebieten wie z.B. den Gesellschaftswissenschaften, den Wirtschaftswissenschaften und vielen anderen Bereichen, die hier in Bezug auf die Didaktik nicht besonders hervorgehoben werden brauchen. S. zu dieser sehr aktuellen Diskussion zwischen den sog. „dogmatischen“ Fächern und den sog. „Grundlagenfächern" die Beiträge von Grundmann, Guttmann, Hillgruber, Lorenz, Rixen und Stolleis in JZ 2013, 693 ff., zum Bericht des Wissenschaftsrats „Perspektiven der Rechtswissenschaft in Deutschland“ vom 9.11.2012 (www.wissenschaftsrat.de/down-load/archiv/2558-12.pdf; 17.12.2013). 
heißt, der Student muss in der Lage sein, das gelernte Wissen an der Lösung eines komplexen Falles umzusetzen.

An diesen Anforderungen hat sich auch die universitäre Lehre auszurichten. Sie hat neben dem Erwerb von Einzelwissen vor allem die Strukturen des Rechts aufzuzeigen, d.h. den „roten Faden“ der juristischen Denkweise zu vermitteln. Dieser kann freilich nur auf dem Boden von Einzelwissen wachsen, so dass Einzelwissen, d.h. reine Rechtskenntnis und Methodensicherheit einander bedingen.

Bislang erfolgt die Lehre an den Rechtsfakultäten im Wesentlichen den klassischen universitären Lehrformen wie Vorlesungen, Übungen, Tutorien, Examinatorien und Seminaren. Allenfalls komplettiert wird diese Art der Lehre durch zusätzliche praktische Übungsmöglichkeiten, wie sie z.B. die immer zahlreicher werdenden simulierten Gerichtsverhandlungen („Moot Courts“) sowie - in ansteigender Tendenz - studentische Rechtsberatung („Law Clinics“) unter Aufsicht und Anleitung durch Lehrende bieten.

Nur diese Präsenzformen gestatten es, Studierende (auch in so genannten Massenvorlesungen) anzuleiten. Ein aufmerksamer Lehrender spürt das feedback der Hörer. Er sieht - wenn er es will - an ihren Blicken und sonstigen Reaktionen, ob seine Botschaft ankommt, und kann flexibel darauf reagieren. Wichtig an diesen klassischen Lehrformen ist natürlich auch der Kontakt zwischen den Studierenden. Dieser fachliche Austausch, der sich am Anfang auf Gespräche etwa am Rande der Vorlesung, auf dem Gang der Bibliothek oder in virtuellen Räumen (facebook) beschränken mag, sich im späteren Verlauf des Studiums aber nicht selten in privat organisierten Lerngruppen konkretisiert, ist besonders wichtig für die im Studium zu erwerbende Fähigkeit zur juristischen Argumentation.

Natürlich lernt der Studierende nicht allein durch die akademische Präsenzlehre, sondern in erheblichem Maße auch durch das Selbststudium. Dabei führt an Lehrbuch, Gesetzeskommentar und Fallübungen kein Weg vorbei. Auch diese Lektüre verlangt eine bestimmte Technik, die sehr individuell ist, und die jeder Studierende zunächst für sich herausfinden muss. Bewährt hat sich z.B. eine Mischung aus Lehrbuchlektüre mit Erstellung von Exzerpten (etwa Karteikarten) und das Üben an Fällen. Der Sinn des Exzerptes besteht dabei weniger in seiner späteren Lektüre, sondern ganz entscheidend in seiner Erstellung. Durch die schriftliche Zusammenfassung des gelesenen Stoffes zwingt sich der Lernende selbst, diesen zu durchdenken und zu strukturieren.

\section{Was kann „das Netz“ dazu beitragen?}

\section{Das Netz als Kommunikationsmittel}

Was „das Netz“ dazu beitragen kann, ist zunächst eine Frage der Definition des Begriffs der „netzbasierten“ Lehre. Sofern man unter netzbasierter Lehre den Einsatz des Internets als Kommunikationsmittel verstehen will, ist sie wohl mittlerweile zur Selbstverständlichkeit geworden: Anstatt Vorlesungsgliederungen, Übungsfälle und 
andere Materialien kosten- und personalaufwendig zu vervielfältigen, können sie „ins Netz“ gestellt werden. Auch kann dem Studierenden der mühevolle Weg in die Bibliothek erspart werden, indem etwa die mit dem jeweiligen Thema verbundene Rechtsprechung ausgewählt und elektronisch verfügbar gemacht wird. Doch bereits hier zeigt sich eine Grundproblematik. Die (auch technisch) einfache Veröffentlichung von Lehrmaterialien „im Netz“ kann zu einer Art „Information-Overkill“ führen: Der Studierende lädt sich Informationen herunter, ohne sie wirklich evaluieren und verarbeiten zu können. Die im Netz zur Verfügung stehenden Materialien sind nicht immer gleicher Qualität. Da können Skripte als pdf-Dateien kursieren, die der Lektor eines juristischen Verlags mit gutem Grund ablehnen würde. Überdies handelt es sich in den allermeisten Fällen lediglich um begleitende Informationen zu Präsenzveranstaltungen, also gerade nicht als Ersatz für das Selbststudium anhand von Lehrbuchlektüre gedacht. Als sinnvoll erwiesen hat es sich auch, Vorlesungen als Podcast aufzuzeichnen und den Studierenden über Portale wie z.B. iTunesU $\mathrm{U}^{2}$ zur Verfügung zu stellen. Schon seit geraumer Zeit geschieht das deutschlandweit von einigen Dozenten, die durchwegs auf ein ebenso großes wie positives Echo gestoßen sind. Rückfragen haben interessanterweise ergeben, dass dies auch nicht zu einer nennenswerten Verminderung studentischer Anwesenheit in den Präsenzveranstaltungen führt. Den Studierenden ermöglicht es neben der Wiederholung des Stoffs zugleich, virtuell Lehrveranstaltungen an anderen Universitäten zu besuchen und damit von Lehrangeboten zu profitieren, die ihnen „live“ nicht zur Verfügung stehen.

Das alles aber ist keine neue Lehrmethode, kein „netzbasiertes Lernen“ im eigentlichen Sinne, sondern lediglich eine mediale Unterstützung und Verbreitung der klassischen Präsenzlehre, die durch die technische Entwicklung der letzten Jahre möglich wurde: Der Podcast kann eben nicht besser sein als die Vorlesung selbst.

\section{MOOCs, Lernvideos und Youtube}

Auch andere Arten netzbasierter Lehre können im Jurastudium allenfalls eine Ergänzungsfunktion haben. Lediglich ergänzende Wirkung dürften hier die seit Neuestem propagierten MOOCs (Massive Open Online Courses) haben. MOOCs sind onlinegestützte Kurse mit relativ kurzen verfilmten Lehreinheiten. Auf diese Weise mag man noch ein juristisches Einzelproblem darstellen können. ${ }^{3}$ Ein Rechtsgebiet in seiner komplexen Einheit kann man sich damit aber nicht erschließen, schon gar nicht die Stellung dieses Rechtsgebiets im Gesamtkontext des nationalen, europäischen und internationalen Rechts. Aber auch in Bezug auf Einzelprobleme können solche Lehrformen allenfalls im Rahmen der Wiederholung des Stoffs einen gewissen Memo-Effekt haben. Ein Jurist lebt aber - im Examen wie in der Praxis - von seinem Wissen und seiner Sprache. Dazu gehört auch das Lesen: Die Generation der „digital natives" wird sich damit abfinden müssen, dass ein Universitätsstudium gerade bei

2 S. dazu beispielhaft das Programm „LMU on iTunes U“ der Ludwig-Maximilians-Universität unter www.uni-muenchen.de/ueber_die_lmu/lmu_on_itunes_u/index.html (17.12.2013).

3 Für Beispiele aus dem Bereich des Strafrechts s. etwa http://iureo.wordpress.com/ (17.12.2013). 
einer Geisteswissenschaft an der Lektüre von Texten und ihrer intellektuellen Verarbeitung nicht vorbeikommt.

Gleiches gilt für die bereits jetzt im Netz vorhandenen Lehrfilme (s. etwa www.telejura.de), in welchen z.B. klassische juristische Entscheidungen, wie etwa der berühmte Gemüseblattfall des BGH, ${ }^{4}$ szenisch dargestellt werden. ${ }^{5}$ Das kann einen Jurastudenten im besten Fall amüsieren - ein Mehrwert beim Lernen ist nicht zu erkennen. Auch elektronische Karteikarten etwa in Gestalt von Lern-Apps für Smartphones, wie sie bereits auf dem Markt vorhanden sind (s. etwa „Nomos-App“), können nur eine sehr geringe ergänzende Funktion haben, wenn sie nicht gar vollständig in den Bereich der „Spielereien“ gehören. Der Lerneffekt der Karteikarte besteht nämlich, wie erwähnt, eher im Verfassen als in der Lektüre. Gänzlich trüb wird das Bild bei Audio-CDs, die in stumpfsinnigster Weise Definitionen abfragen. ${ }^{6}$ Wer hierdurch Jura lernen will, kann nur depressiv werden: Gähnend langweiliges Vorlesen aus schlechten Repetitorskripten ist sicherlich kein e-learningKonzept. Der Anspruch dieser Produkte an sich selbst ist offenbar nicht übermäßig hoch, wenn man sieht, mit welchem Benutzerfeedback hier auch noch geworben wird: „Ich habe in Grundrechte keine einzige Vorlesung besucht und keine AG. Als dann der Stoff auf einige Artikel eingeschränkt wurde, habe ich mir lediglich unmittelbar vor der Klausur immer und immer wieder die Standardfälle dazu angehört. Das Ergebnis waren 6 Punkte. Wenn ich jetzt noch zusätzlich Skripte und andere Lektüre bearbeitet hätte, wären es vermutlich mehr Punkte geworden, aber ich wollte sowieso nur bestehen. “7 Wer mit diesem Anspruch Jura „studiert“, hat nichts Besseres verdient. Denn der oben beschriebene immense Lerneffekt des eigenen Erstellens von Exzerpten (z.B. auf Karteikarten) wird dabei vollständig versäumt. Mit den dort etwa verbreiteten Frage-Antwort-Spielen juristischer Definitionen wird mit Sicherheit keine einzige Examensklausur bestanden. Auch die derzeit ebenfalls vieldiskutierte Lehrmethode des „serious gaming“ kann im Jurastudium kaum sinnvoll eingesetzt werden. Beim Lernen dürfte es sich eher als Zeiträuber erweisen. ${ }^{8}$

\section{Die „Reps“ als Nagelprobe}

Nun setzt sich ein Hochschullehrer der Rechtswissenschaften mit einem eher konservativen Lehransatz nahezu automatisch dem Vorwurf der Innovationsfeindlichkeit aus. Da lohnt sich ein Blick in die „Tabuzone“: Was machen eigentlich die kommerziellen Repetitorien? Dort regiert bekanntlich der Markt, der ja - zumindest in diesem Bereich - automatisch dafür sorgt, dass sich das bessere Produkt durchsetzt. Auch die Repetitorien setzen aber erkennbar weiter auf Präsenzunterricht. Dieser findet durch das Netz allenfalls weitere kommerzielle Verbreitung, ohne dass man

4 BGHZ 66, 51.

5 S. www.youtube.com/watch?feature=player_embedded \&v=AEIqItxor7w (17.12.2013).

6 S. dazu als „Horrorbeispiel“ die Produktpalette von „Niederle Media“ unter www.niederle-media.de (17.12.2013).

7 www.niederle-media.de/Urteile-der-Leser (17.12.2013).

8 S. etwa www.juraquiz.de (17.12.2013). 